\title{
MULTIPLEX ELECTIONIS METHODUS (MEM) APLICADO A UM PROBLEMA DE SELEÇÃO DUPLA
}

\author{
Henrique Rego Monteiro da Hora \\ Instituto Federal de Educação, Ciência e Tecnologia Fluminense - IFF \\ henrique.dahora@iff.edu.br \\ Helder Gomes Costa \\ Universidade Federal Fluminense ï UFF \\ hgc@pq.cnpq.br
}

\begin{abstract}
Resumo
O problema de decisão múltipla já é conhecido na teoria da decisão, sendo normalmente dividido em problemas mais simples para se adequarem à métodos tradicionais. A comunidade científica já proveu o MEG, que propõe uma modelagem inicial para este tipo problema. Este trabalho aplica o Multiplex Electionis Methodus ao mesmo problema resolvido pelo MEG a fim de gerar comparações entre o resultado do MEM e de uma ordenação por média ponderada. Apresenta-se também as melhorias do algoritmo e ao final constata-se que as duas primeiras alternativas de uma ordenação não compõem necessariamente o melhor grupo de $\mathrm{n}$ alternativas.
\end{abstract}

Palavras-chave: Decisão Múltipla, Multicritério, MEG, MEM.

\begin{abstract}
The multiple decision problem is already known in the decision theory, being usually divided into simpler problems to fit the traditional methods. The scientific community has provided the MEG, which proposes an initial model for this type of problem. This research applies the Multiplex Electionis Methodus on the same problem solved by MEG in order to generate comparisons between the results of the MEM and of a ranking by weighted mean. Moreover, the improvements on the algorithms are also presented, and the end it is observed that the first two alternatives of a ranking are not necessarily the best group of $\mathrm{n}$ alternatives.
\end{abstract} Key-words: Multiple Decision, Multicriteria, MEG, MEM. 


\section{INTRODUÇÃO}

Os problemas de decisões múltiplas, apesar de já apontados há algum tempo por Vincke (1992), passaram por lapso temporal sem tratamento analítico específico. Este tipo de problema assemelha-se a uma problemática de portfólio (BELTON; STEWART, 2002), derivada da problemática de escolha (P.alfa) definida por Roy e Bouyssou (1993), diferindose apenas na definição $a$ priori do tamanho do subgrupo a ser escolhido.

Vincke (1992) apresenta está problemática pelo exemplo de localização de duas usinas hidroelétricas, que como trabalharam em conjunto para prover energia em um mesmo sistema, e sugere que a solução deve considerar a combinação das alternativas disponíveis.

Em geral, o tratamento dado a este tipo de problema se divide em duas abordagens: (i) utilizar um método para ordenação de alternativas e escolher os dois melhores posicionados, ou (ii) escolher a melhor alternativa de uma primeira ordenação e retirá-la do conjunto de alternativas; redistribuir as ponderações dos critérios, uma vez que os critérios já foram contemplados em algum grau pela primeira escolha, gerar uma nova ordenação, e selecionar a melhor opção desta segunda ordenação, dividindo, assim, o problema assim em duas etapas.

Não há certeza nem que a situação (i) seja a melhor, nem que a situação (ii) o seja, pois não há garantia que esta escolha das duas melhores alternativas individualmente seja necessariamente o melhor conjunto de duas alternativas.

Hora e colaboradores (2012) e Hora e Costa (2013) propuseram uma modelagem à luz da teoria multicritério que resolvesse esta situação, e propuseram o MEG - Modus Eligendi Geminus, ou método de seleção dupla em vernáculo, que apresenta uma solução mais satisfatória do problema de seleção múltipla, contudo, apresentava algumas limitações, como necessidade de uma mesma escala de medida para todos os critérios, só ser capaz de medir em um sentido de preferência e ser limitado a somente seleções de duas alternativas concomitantemente.

Este trabalho apresenta o MEM - Multiplex electionis methodus, ou método de seleção dupla, que e ilustra sua aplicação em um problema de seleção dupla por meio de um software homônimo desenvolvido para implementação de seu algoritmo. Tanto o software, quanto seu código fonte, estão disponíveis em http://www.github.com/MCDALab/MEM (Figura 1).

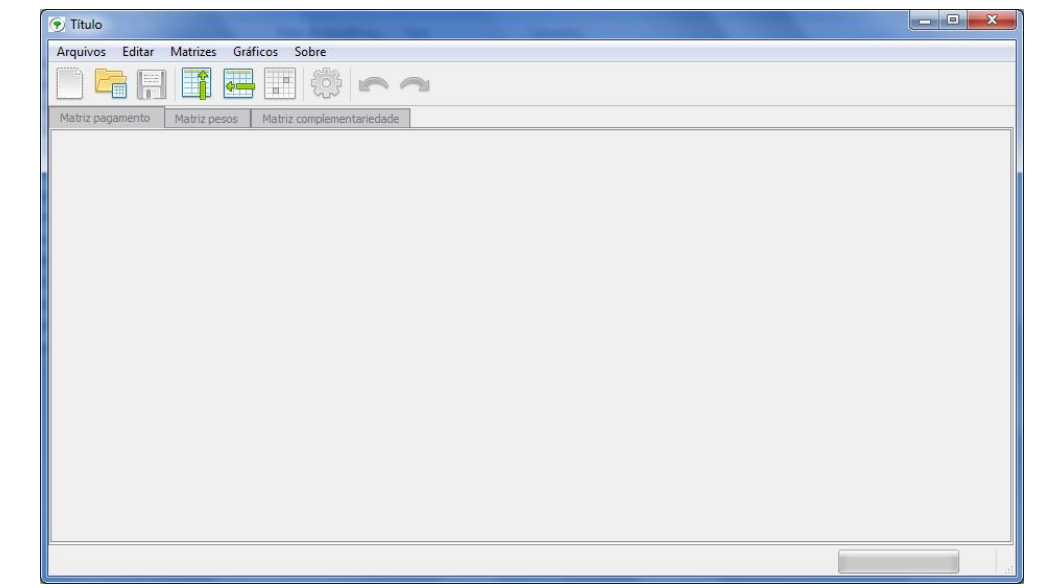

Figura 1: Interface do software homônimo que implementa o algoritmo do MEM.

Além do método proposto, espera-se com a disponibilização do software sob licença livre, habilitar qualquer pesquisador e/ou profissional em utilizar plenamente o algoritmo, mesmo sem ser capaz de implementar seus procedimentos, mas apenas por meio da interface do software. 


\section{ABORDAGEM METODOLÓGICA}

Para ilustração do método e utilização do software desenvolvido, utiliza-se os mesmos dados utilizados no MEG em Hora et al. (2012), com o mesmo objetivo de selecionar duas alternativas concomitantemente. $\mathrm{O}$ trabalho não se ocupará em apresentar todas as matrizes utilizadas utilização do método, uma vez que já estão publicamente disponíveis em Hora e colaboradores (2012) e em Hora e Costa (2013). Dar-se-á preferência a explorar os resultados neste trabalho.

Os resultados são analisados comparando a seleção intuitiva das duas alternativas melhores ranqueadas em um procedimento de média ponderada, e os resultados obtidos pelo MEM.

Para verificação da estabilidade do resultado foi realizada uma análise de sensibilidade (TANINO, 1999) por meio da correlação rho de Spearman para comparar os diferentes resultados achados pela variação dos parâmetros do problema. Esta técnica foi empregada, por exemplo, em Brans; Vincke; Mareschal (1986) para comparação entre as ordenações obtidas pelo PROMETHEE e ELECTRE III.

A correlação de Spearman, recomendada para medir a relação entre duas variáveis de uma escala ordinal (GRAVETTER; WALLNAU, 2008) foi obtida pelo software SPSS 13, que produziu também os resultados gráficos.

O coeficiente de correlação de postos de Spearman, denotado por $r_{\mathrm{s}}$, é simplesmente o rho de Pearson calculado para os postos dos valores de $x$ e $y$ (Equação 1). Portanto:

$$
r_{s}=\frac{\sum_{i=1}^{n}\left(x_{r i}-\bar{x}_{r}\right) \cdot\left(y_{r i}-\bar{y}_{r}\right)}{\sqrt{\left[\sum_{i=1}^{n}\left(x_{r i}-\bar{x}_{r}\right)^{2}\right] \cdot\left[\sum_{i=1}^{n}\left(y_{r i}-\bar{y}_{r}\right)^{2}\right]}}
$$

Equação 1: Coeficiente de correlação rho de Spearman (PAGANO; GAUVREAU, 2004).

Onde $x_{r i}$ e $y_{r i}$ são os postos associados com o $i$-ésimo indivíduo em vez das observações reais.

Analisa-se também a situação para verificar a efetividade do modelo executando-se os procedimentos do algoritmo com uma matriz de complementaridade dos critérios complementares, chamada de matriz K que é obtida pelo valor complementar para se chegar a 1 (um), da matriz C, conforme verificado na Equação 2.

$$
k_{j j^{\prime}}=\left|1-c_{j j^{\prime}}\right|
$$

Equação 2: Determinação do valor da matriz K para análise da robustez do MEM.

Por fim, procura-se identificar a alternativa mais complementável, para a situação de ter que escolher somente uma no momento, mas outra futuramente, por meio da análise da frequência de cada alternativa nas dez primeiras posições da ordenação da escolha dupla.

\section{BREVE DESCRIÇÃO DO MÉTODO}

$\mathrm{Na}$ resolução de problemas multicritério, as inspirações para sua solução podem advir por meio de uma matemática não mais sofisticada do que a álgebra do ensino médio (high school) em detrimento do uso de matemática avançada. A verdade é que quanto mais simples a ferramenta analítica, melhor (RAIFFA, 2002). O método brasileiro TODIM, por exemplo, teve seu desenvolvimento norteado para se obter uma ferramenta de tomada de decisão que pudesse ser utilizada por qualquer um que tivesse conhecimento básico de álgebra linear (GOMES; LIMA, 1991).

Corroborando com isto, a simplicidade e a interatividade devem ser as linhas de 
força da atividade de apoio à decisão (BANA E COSTA, 1993). Vargas (1990) atribui o sucesso do AHP como conseqüência da sua simplicidade e robustez.

Vincke (1992) coloca que em um processo decisório, a escolha de uma alternativa concomitante com outra gera um novo conjunto resultante das várias combinações possíveis deste conjunto inicial, gerando assim um conjunto finito de soluções viáveis advindas da teoria de otimização combinatória (EHRGOTT, 2005).

A proposta deste trabalho está em, a partir de uma avaliação individual das alternativas à luz dos critérios, e mensuração da sinergia entre eles, proceder com a escolha de ónôalternativas, de acordo com a necessidade ou desejo do decisor.

O método proposto pode ser apresentado em cinco passos:

1. Elaborar a matriz $\mathrm{A}_{\mathrm{i}, \mathrm{j}}$ de pagamentos das ñò alternativas a luz dos ñjò critérios;

2. Elaborar a matriz $\mathrm{C}_{\mathrm{j}, \mathrm{j}^{\prime}}$ de critérios complementares, registrando o relacionamento interno entre os critérios;

3. Elaborar uma matriz linha (vetor) $\mathrm{W}_{\mathrm{j}}$, contendo os pesos de cada critério, para definição do perfil da escolha;

4. Estabelecer o valor do tau (Ü) de corte;

5. Calcular a matriz $\mathrm{B}_{1, \mathrm{~m}}$, combinando os critérios dois a dois, e combinando as alternativas, tomadas 'n' a 'n', ambos sem repetição, levando em consideração valor do tau (Ü) corte.

\subsection{Elaborar A MATRIZ DE PAGAMENTOS}

A fase matriz de pagamentos dá-se de forma tradicional aos dos métodos da escola européia (FIGUEIRA; MOUSSEAU; ROY, 2005), aonde cada alternativa é avaliada à luz de um conjunto de critérios.

Atribui-se o nome de Matriz A para a matriz de pagamentos, cuja avaliação de cada critério deve ser feita na própria escala de mensuração. A Matriz A deve ser normalizada segundo a Equação 3, de modo a deixar todas as avaliações na mesma escala e com a mesma direção de preferência.

$$
a_{i j}^{\prime}=\frac{\left|a_{i j}-\operatorname{pior}\left(a_{j}\right)\right|}{\left|\operatorname{melhor}\left(a_{j}\right)-\operatorname{pior}\left(a_{j}\right)\right|}
$$

Equação 3: Normalização da matriz de pagamentos.

\subsection{ELABORAR A MATRIZ' DE CRITÉRIOS COMPLEMENTARES}

A matriz de critérios complementares, chamada no método de Matriz $\mathrm{C}$ vem mensurar o quanto um critério influencia outro (TIETZ, 1992), ou nas palavras de Almeida e Duarte (2011) medem a sinergia de cooperação entre as partes envolvidas.

Utiliza-se uma escala monotônica, incrementada em 0,1 , de zero a 1 , no qual se faz a avaliação entre dois critérios segundo a classificação presente no Quadro 1.

\begin{tabular}{|l|l|}
\hline Classificação & \multicolumn{1}{|c|}{ Definição } \\
\hline Comp. Moderada & $\begin{array}{l}\text { Forte complementação (entre 0,6 e 1,0) - quando o critério se relaciona } \\
\text { muito pouco, tem escassos temas em comum, e não há nenhuma } \\
\text { dependência }\end{array}$ \\
\hline $\begin{array}{l}\text { Complementação moderada (entre } 0,4 \text { e } 0,6 \text {, inclusos) - quando os } \\
\text { critérios têm relacionamento mediano, existindo alguns temas em } \\
\text { comum; }\end{array}$
\end{tabular}




\begin{tabular}{|l|l|}
\hline Pouca comp. & $\begin{array}{l}\text { Pouca complementação (entre 0,1 e 0,4) - quando os critérios têm } \\
\text { grande relacionamento, vários temas em comum e há dependência entre } \\
\text { eles. }\end{array}$
\end{tabular}

Quadro 1: Descrição da complementação entre critérios.

\subsection{ELABORAR VETOR DE PESOS}

Um problema fundamental na teoria da decisão é como atribuir pesos a um conjunto de critérios de acordo com suas importâncias (SAATY, 1977). A importância relativa dos critérios é uma parte crucial de informação.

É sabido que os critérios não têm sempre a mesma importância, então é útil definir um peso $\left(\mathrm{W}_{\mathrm{i}}\right)$ associado a cada critério 'i'. A distribuição de pesos a esses de modo a representar a sua importância relativa é sempre um ponto delicado (ROGERS; BRUEN; MAYSTRE, 2000). Tais pesos representam a força ou a importância deste critério (MARICHAL; ROUBENS, 2000).

\subsection{ESTABELECER O VALOR DO TAU DE CORTE}

O tau (Û̉ de corte é uma contribuição da abordagem descritiva da tomada de decisão, que ao invés de se trabalhar com a maximização de resultados, afirma que uma solução eleita pode ser somente ñsuficientemente satisfatóriaò (FAÇANHA; YU, 2011), ou seja, uma otimização precisa não é necessária, sendo suficiente estar próximo à esta otimização (DEMING, 1990), admitindo elementos de limitação da racionalidade do decisor e balanceando o modelo (TIETZ, 1992).

Pelo modelo do MEM adotar sucessivas multiplicações de números entre 0 (zero) e 1 (um) em seus mecanismos internos, sua determinação deve ser cuidadosa e não figurar próximo a 1.

\subsection{CALCUlar A MATRIZ Final}

A Matriz B pode ser encarada como uma matriz de pagamentos para problemas combinatórios. Isto por que tanto o número de alternativas e critérios são determinados a partir da combinação $\mathrm{n}$ a $\mathrm{n}$ da matriz de pagamentos original (Matriz A). A Figura 2 ilustra este procedimento.

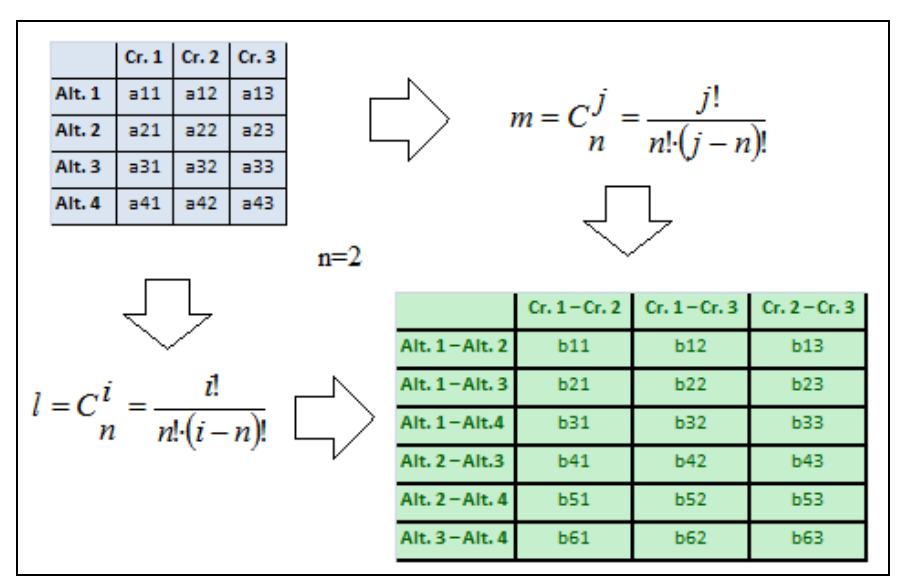

Figura 2: Ilustração da elaboração da Matriz B.

Por meio da combinação dos critérios tomados dois a dois (sendo dois o número de escolhas desejado), e da combinação das alternativas tomadas também dois a dois, chega-se a uma nova matriz de pagamentos, cujo valor dos itens deve ser determinado segundo a Equação 4. 


$$
b^{\prime}{ }_{l m}=\frac{\sum_{i} \sum_{j} a_{i j}^{\prime}}{n^{2}} \cdot \frac{\sum_{j} w_{j}}{n} \cdot c^{\prime}, \forall i \in L, \forall j \in M
$$

Equação 4: Determinação dos elementos da Matriz Bô

Onde a $\hat{Q}_{\mathrm{j}}$ são os elementos da Matriz AQ̂ $\mathrm{w}_{\mathrm{j}}$ os elementos do vetor de pesos W e é'ôé a média dos índices de complementaridade entre critérios recuperados a partir dos elementos da Matriz C de complementaridade entre critérios.

Por fim, o tau de corte é aplicado conforme ilustrado na Equação 5 e determinação final da matriz B.

$$
b_{l m}=\left\{\begin{array}{l}
1 \rightarrow b_{l m}^{\prime} \geq \tau \\
0 \rightarrow b^{\prime}{ }_{l m}<\tau
\end{array}\right.
$$

Equação 5: Aplicação do tau de corte.

Assim, o maior valor obtido pela Equação 6 é considerado a melhor (ou melhores) alternativa(s), onde o número de alternativas varia de acordo com o valor de corte (Û́ aplicada no método.

$$
\frac{\sum_{m=1}^{k} b_{l m}}{k}, \forall l
$$

Equação 6: Função aditiva para determinação do índice para escolha do melhor conjunto de alternativas.

Cabe a esta equação, apresentar os valores obtidos que satisfaçam a função (valor) corte por meio do somatório para cada conjunto de alternativa. Em outras palavras, a Equação 6 tem por finalidade indicar a quantidade de resultados, de cada possibilidade de escolha, que forem iguais ou maiores que o valor do tau (Ü) de corte. Este último procedimento descaracteriza o método como compensatório.

\section{RESULTADOS}

O resultado intuitivo é a escolha das alternativas A8 e A5, por estas figurarem nas posições 1 e 2 da ordenação por média ponderada (HORA et al., 2012; HORA; COSTA, 2013). Contudo, ao utilizar o MEM e considerar a complementaridade entre critérios, alcançase a quantidade de 45 alternativas (dez alternativas tomadas dois a dois) e 153 critérios (18 critérios, também tomados dois a dois), que resultam em uma Matriz B com 6.885 avaliações.

O resultado do MEM para escolha dupla é apresentado no Quadro 1, onde a coluna

\begin{tabular}{|c|c|c|c|c|c|c|c|c|c|c|c|c|c|c|}
\hline Ord. & Alt. & $\overline{\%}$ & Ord. & Alt. & $\%$ & Ord. & Alt. & $\overline{\%}$ & Ord. & Alt. & $\%$ & Ord. & Alt. & $\%$ \\
\hline $1 .^{\circ}$ & $\mathrm{A} 1, \mathrm{~A} 2$ & 70,5 & $10^{\circ}$ & $\mathrm{A} 3, \mathrm{~A} 8$ & 57,3 & $19 .^{\circ}$ & $\mathrm{A} 3, \mathrm{~A} 4$ & 54,2 & $28 .^{\circ}$ & A1, A6 & 46,4 & $37 .^{\circ}$ & $\mathrm{A} 3, \mathrm{~A} 10$ & 39,8 \\
\hline $1 .^{\circ}$ & A7, A8 & 70,5 & $11 .^{\circ}$ & $\mathrm{A} 1, \mathrm{~A} 8$ & 66,0 & $20 .^{\circ}$ & $\mathrm{A} 2, \mathrm{~A} 4$ & 51,6 & $29^{\circ}$ & A2, A9 & 45,7 & $38 .^{\circ}$ & $\mathrm{A} 1, \mathrm{~A} 10$ & 39,2 \\
\hline $3 .^{\circ}$ & $\mathrm{A} 2, \mathrm{~A} 3$ & 69,9 & $11^{\circ}$ & $3, \mathrm{~A} 5$ & 66,0 & $200^{\circ}$ & $\mathrm{A} 8, \mathrm{~A} 10$ & 51,6 & $29 .^{\circ}$ & A3, A9 & 45,7 & $39 .^{\circ}$ & $2, \mathrm{~A} 10$ & 36,6 \\
\hline $3 .^{\circ}$ & A7 & 9,9 & $11^{\circ}$ & 48 & ,, 0 & 22 & 87 & 50,9 & $29 .^{\circ}$ & A6 & 45,7 & $40 .^{\circ}$ & A6 & 32,0 \\
\hline $3 .^{\circ}$ & $\mathrm{A} 3, \mathrm{~A} 7$ & 69,9 & $14 .^{\circ}$ & $\mathrm{A} 1, \mathrm{~A} 3$ & 65,3 & $23 .^{\circ}$ & $\mathrm{A} 1, \mathrm{~A} 4$ & 50,3 & $29 .^{\circ}$ & A5, A9 & 45,7 & $41 .^{\circ}$ & $\mathrm{A} 4, \mathrm{~A} 9$ & 28,7 \\
\hline $6^{\circ}$ & $\mathrm{A} 1, \mathrm{~A} 7$ & 69,2 & $15^{\circ}$ & $\mathrm{A} 1, \mathrm{~A} 5$ & 64,7 & $24 .^{\circ}$ & A6, A8 & 49,0 & $29 .^{\circ}$ & $\mathrm{A} 5, \mathrm{~A} 10$ & 45,7 & $41 .^{\circ}$ & $\mathrm{A} 4, \mathrm{~A} 10$ & 28,7 \\
\hline $6^{\circ}$ & $\mathrm{A} 2, \mathrm{~A} 8$ & 69,2 & $16^{\circ}$ & $\mathrm{A} 4, \mathrm{~A} 5$ & 55,5 & $25 .^{\circ}$ & A3, A6 & 47,7 & $29 .^{\circ}$ & $\mathrm{A} 7, \mathrm{~A} 10$ & 45,7 & $43 .^{\circ}$ & $\mathrm{A} 6, \mathrm{~A} 10$ & 26,8 \\
\hline $7 .^{\circ}$ & $\mathrm{A} 2, \mathrm{~A} 5$ & 68,6 & $16^{\circ}$ & $\mathrm{A} 4, \mathrm{~A} 7$ & 55,5 & $25^{\circ}$ & A7, A9 & 47,7 & $29 .^{\circ}$ & A9 & 45,7 & $44 .^{\circ}$ & A9 & 20,9 \\
\hline $7 .^{\circ}$ & A5, A7 & 68,6 & $18 .^{\circ}$ & $\mathrm{A} 4, \mathrm{~A} 8$ & 54,9 & $27 .^{\circ}$ & A2, A6 & 47,0 & $36^{\circ}$ & A1, A9 & 43,7 & $45 .^{\circ}$ & A9, A10 & 16,9 \\
\hline
\end{tabular}
"Ord." ilustra a posição da ordenação, "Alt." indica a alternativa e "\%" indica em a porcentagem dos 153 critérios combinados que superaram o tau de corte.

$\mathrm{O}$ resultado apresentado indica as alternativas $\{\mathrm{A} 1 ; \mathrm{A} 2\}$ e $\{\mathrm{A} 7 ; \mathrm{A} 8\}$ como sendo os melhores conjuntos de duas alternativas, dado os critérios considerados e também a interação 
entre eles. Esta situação de apontar mais de uma solução como a melhor possível está de acordo com a teoria multicritério, uma vez que não se pretende ñindicar ao decisor uma solução única e verdadeiraò(GOMES; GONZÁLEZ-ARAYA; CARIGNANO, 2004).

Ao testar a estabilidade do modelo, pela a correlação de Spearman, obtém-se uma forte correlação entre os resultados, abalizando, assim, a robustez dos resultados.

\begin{tabular}{cl|c|c|c} 
& & $\mathbf{t a u}=\mathbf{0 , 2 0}$ & $\mathbf{t a u}=\mathbf{0 , 1 9}$ & $\mathbf{t a u = 0 , 2 1}$ \\
\hline tau=0,20 & Coef. De Correlação & 1,000 & 0,983 & 0,983 \\
& Sig. (bi-caudal) &. & 0 & 0 \\
& N & 45 & 45 & 45 \\
\hline tau=0,19 & Coef. De Correlação & 0,983 & 1,000 & 0,966 \\
& Sig. (bi-caudal) & 0 & $\cdot$ & 0 \\
& N & 45 & 45 & 45 \\
\hline tau=0,21 & Coef. De Correlação & 0,983 & 0,966 & 1,000 \\
& Sig. (bi-caudal) & 0 & 0 &. \\
& N & 45 & 45 & 45 \\
\hline
\end{tabular}

Quadro 2: Resultado da correlação de Spearman para a análise de sensibilidade.

No Quadro 2 verifica-se uma correlação sempre forte entre os experimentos realizados: o tau de corte igual a 0,20 apresentou uma correlação de 0,983 com o com o tau igual à 0,19 e 0,983 com o tau de 0,21 .

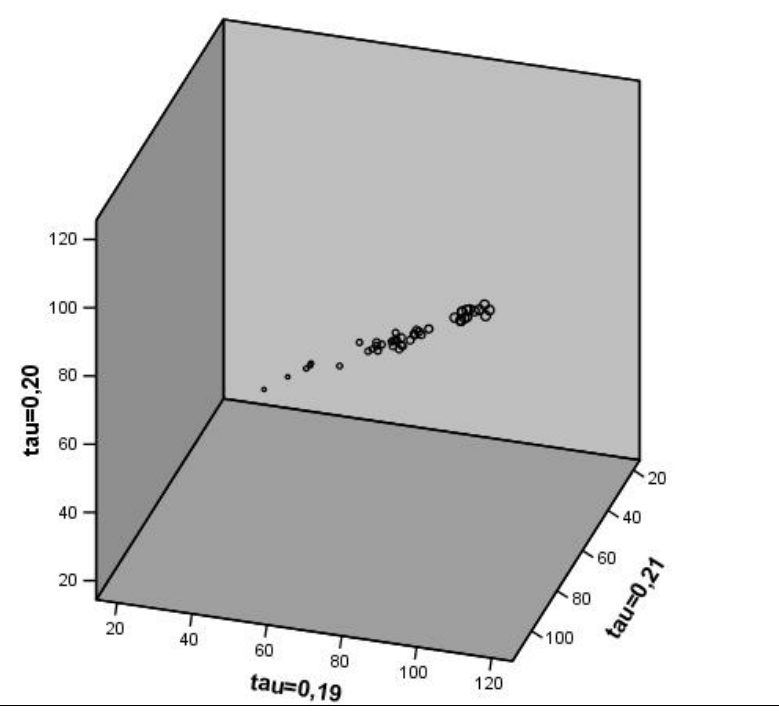

Figura 3: Gráfico da correlação de Spearman da análise de sensibilidade.

No gráfico da Figura 3verifica-se a alta correlação entre os experimentos realizados pela tendência dos dados em torno de uma reta imaginária entre eles, ilustrando o elevado coeficiente de correlação apresentado no Quadro 2. Já o gráfico da Figura 4 apresenta uma análise da variação do tau de corte de 0,00 até 1,00 com variação de 0,01 .

No eixo primário das ordenadas, é plotada a variação da porcentagem que a(s) primeira(s) alternativa(s) alcança(m), podendo variar de 0 até 1 (onde 1 equivale a 100\%). No eixo secundário das ordenadas, é plotada a quantidade de alternativas que possuem esta melhor marca para este tau de corte, podendo neste caso variar de 0 (zero) - nenhum caso foi observado, até 45 (10 alternativas iniciais, tomadas duas a duas, sem repetição). Por último, no eixo das abscissas têm-se a variação do tau de 0 até 1 , com incremento de 0,01 . 


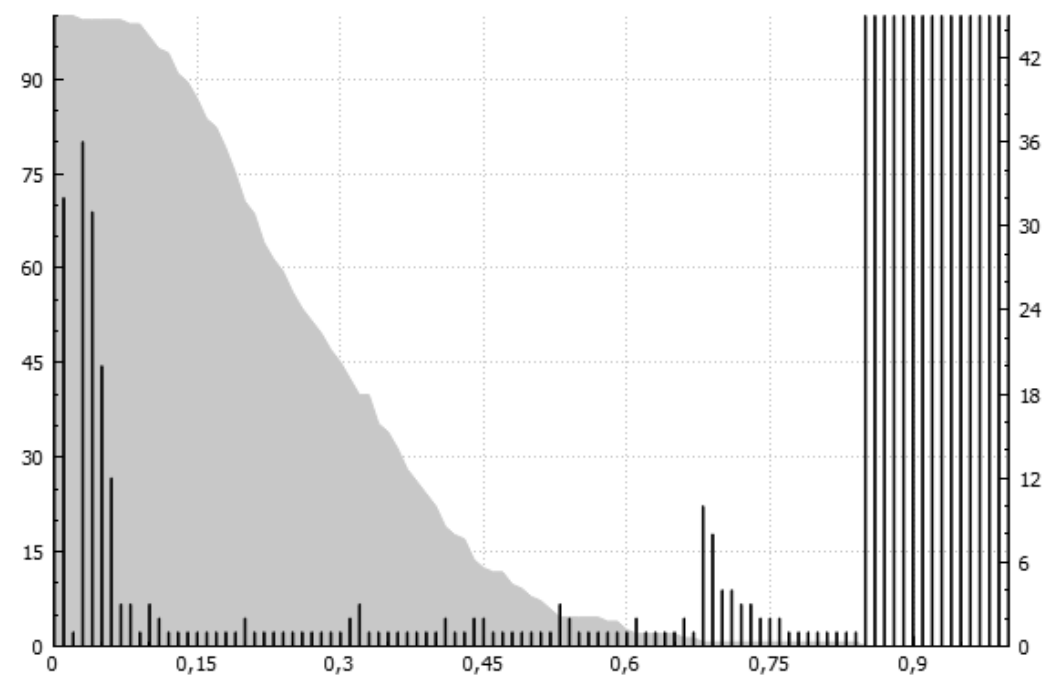

Figura 4: Análise de sensibilidade do tau de corte para o problema de escolha dupla.

Pode ser verificado na Figura 4 que a região que produz uma escolha cuja porcentagem de superação figura próximo aos desejados $80 \%$ está cerca do valor arbitrário $0,2(70,59 \%)$.

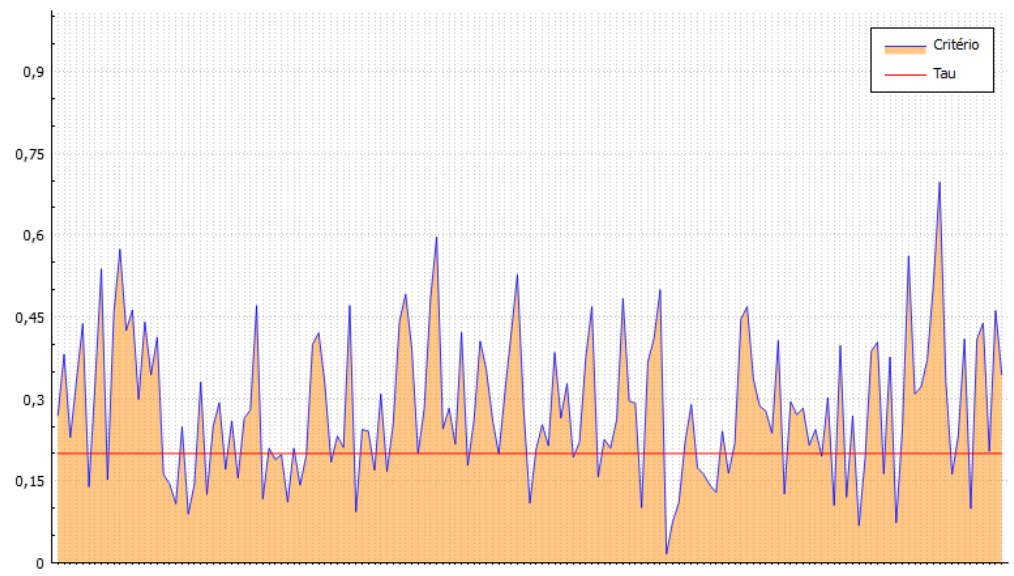

Figura 5: Gráfico ilustrativo da aplicação do tau de corte da alternativa $\{$ A7, A8\}.

A Figura 5 contempla um gráfico com duas séries. A série apresentada em área ilustra os valores da Matriz B', isto é, os valores antes da aplicação do tau de corte, enquanto a série pontilhada indica onde há valores que superam o tau de corte, presentes na Matriz B (pontos na linha 0,2 ) e onde não há superação do tau. $\mathrm{O}$ eixo das abscissas representa os critérios das matrizes B e B', totalizando 153 pontos (18 critérios tomados dois a dois), e o eixo das ordenadas o desempenho destes, tendo o mínimo valor possível em 0 (zero) e o máximo valor possível em 1 (um).

Esta ilustração evidencia o caráter não compensatório do método, onde qualquer item acima do tau de corte pontua igualmente, superando minimamente ou largamente. 


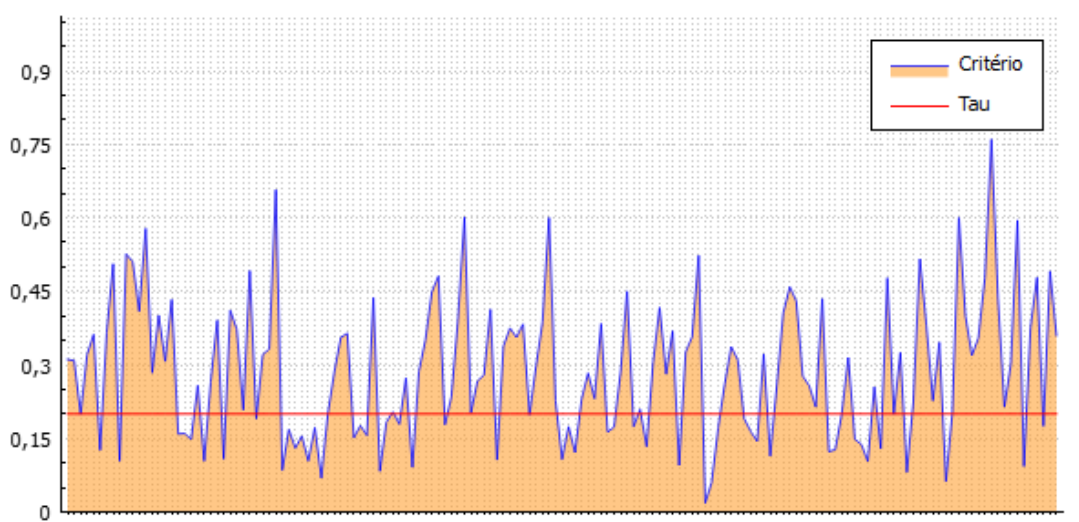

Figura 6: Gráfico ilustrativo da aplicação do tau de corte da alternativa $\{\mathrm{A5}, \mathrm{A8}\}$.

Na Figura 6 ilustra-se a alternativa $\{$ A5; A8 $\}$ que seria a escolha intuitiva pela ordenação resultante do método da média ponderada. Observa-se que há regiões em que a combinação entre desempenho de alternativas, pesos das mesmas, e o índice de complementaridade não são suficientes para superar o tau de corte.

Na Figura 7 apresenta-se para fins comparativos o desempenho da pior alternativa \{A9; A10\}: em poucas ocasiões (somente 26 em 153) há superação do tau de corte.

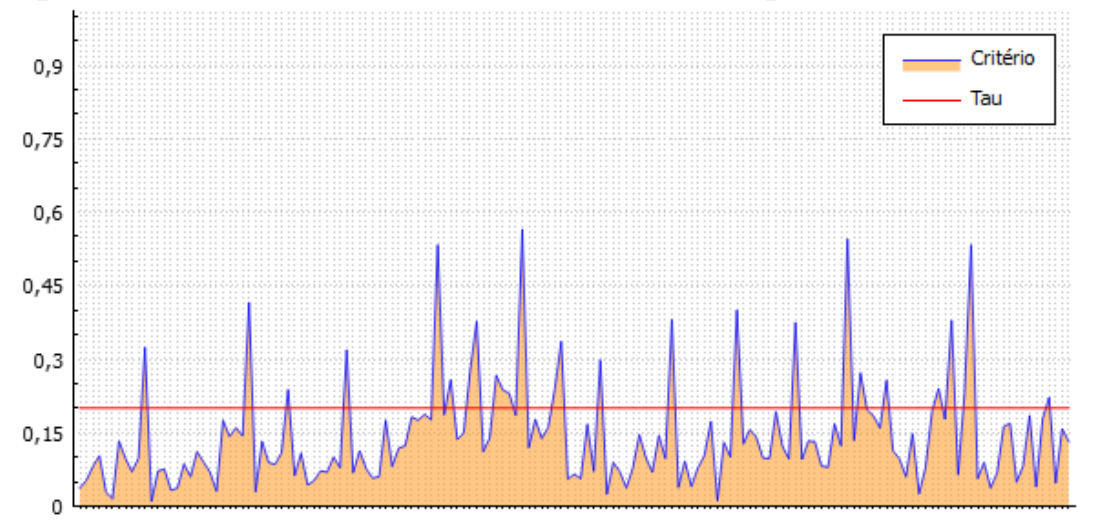

Figura 7: Gráfico ilustrativo da aplicação do tau de corte da alternativa \{A9, A10\}.

No Quadro 3, verifica-se o resultado da aplicação do MEM, substituindo-se a Matriz C pela Matriz K dos complementares da primeira, e verifica-se o problema de inversão de ordem, aonde as primeiras escolhas da aplicação numérica para uma escolha dupla ( $\{\mathrm{A} 1 ; \mathrm{A} 2\}$ e $\{$ A7; A8 \}) figuram somente nas posições 21 e 13 respectivamente.

\begin{tabular}{|c|c|c|c|c|c|c|c|c|c|c|c|c|c|c|}
\hline Ord. & Alt. & \%. & Ord. & Alt. & \% & Ord. & Alt. & \% & Ord. & Alt. & \% & Ord. & Alt. & \% \\
\hline 1 & A2; A5 & 80,4 & 10 & A1; A7 & 77,8 & 17 & A6; A8 & 75,2 & 28 & A7; A9 & 72,5 & 37 & A6; A7 & 70,6 \\
\hline 2 & A2; A3 & 79,7 & 10 & A3; A7 & 77,8 & 17 & A8; A9 & 75,2 & 29 & A2; A6 & 71,9 & 38 & A1; A10 & 69,3 \\
\hline 2 & A2; A8 & 79,7 & 10 & A3; A8 & 77,8 & 21 & A1; A2 & 74,5 & 29 & A2; A9 & 71,9 & 39 & A2; A10 & 68,6 \\
\hline 2 & A5; A8 & 79,7 & 13 & A4; A8 & 77,1 & 22 & A2; A4 & 73,9 & 29 & A3; A10 & 71,9 & 40 & A4; A6 & 68,0 \\
\hline 5 & A1; A5 & 79,1 & 13 & A5; A7 & 77,1 & 22 & A4; A7 & 73,9 & 29 & A7; A10 & 71,9 & 40 & A4; A10 & 68,0 \\
\hline 6 & A1; A3 & 78,4 & 13 & A7; A8 & 77,1 & 22 & A5; A6 & 73,9 & 29 & A8; A10 & 71,9 & 42 & A4; A9 & 67,3 \\
\hline 6 & A1; A8 & 78,4 & 16 & A4; A5 & 75,8 & 22 & A5; A9 & 73,9 & 34 & A1; A9 & 71,2 & 43 & A6; A9 & 61,4 \\
\hline 6 & A2; A7 & 78,4 & 17 & A1; A4 & 75,2 & 26 & A1; A6 & 73,2 & 34 & A3; A9 & 71,2 & 43 & A6; A10 & 61,4 \\
\hline 6 & A3; A5 & 78,4 & 17 & A3; A4 & 75,2 & 26 & A3; A6 & 73,2 & 34 & A5; A10 & 71,2 & 45 & A9; A10 & 5,2 \\
\hline
\end{tabular}

Quadro 3: Resultado da ordenação para três escolhas utilizando a matriz $K$ ao invés da matriz C (Ü=0,04).

Este resultado é corroborado pela análise da correlação de Spearman, cujo resultado é apresentado no Quadro 4. O valor de 0,674 ilustra que houve diversas trocas de posições entre o experimento com a Matriz $\mathrm{C}$ e o experimento com a Matriz K, evidenciando a participação efetiva da Matriz C no modelo desenvolvido.

\begin{tabular}{|l|l|} 
Matriz K & Matriz C \\
\hline
\end{tabular} 


\begin{tabular}{cl|c|c} 
Matriz K & Coef. de Correlação & 1,000 & 0,674 \\
& Sig. (bi-caudal) &. & 0 \\
& N & 45 & 45 \\
\hline Matriz C & Coef. de Correlação & 0,674 & 1,000 \\
& Sig. (bi-caudal) & 0 &. \\
N & 45 & 45 \\
\hline
\end{tabular}

Quadro 4: Resultado da correlação de Spearman para dados obtidos entre a Matriz C e a Matriz K.

É possível adicionalmente, além de escolher o melhor conjunto de duas alternativas, buscar a melhor escolha que possui o melhor potencial de complementaridade, isto é, por ocasião de uma única escolha e posteriormente de uma segunda, é possível apontar uma alternativa com potencial de mais facilmente complementar às demais.

Considerando o conjunto das dez primeiras alternativas apresentadas no Quadro 1, as seguintes frequências são obtidas.

Tabela 1: Frequência das alternativas entre as dez primeiras escolhas.

\begin{tabular}{|c|c|c|c|c|}
\hline \multirow{2}{*}{ Alt. } & \multicolumn{3}{|c|}{ Frequência } & \\
\hline & $\hat{\mathbf{U}}=0,20$ & $\hat{\mathbf{U}}=\mathbf{0 , 2 1}$ & $\hat{\mathbf{u}}=0,19$ & \\
\hline $\mathrm{A} 2$ & 5 & 5 & 5 & 15 \\
\hline A7 & 5 & 5 & 5 & 15 \\
\hline A8 & 3 & 3 & 3 & 9 \\
\hline A3 & 3 & 3 & 3 & 9 \\
\hline A1 & 2 & 2 & 2 & 6 \\
\hline A5 & 2 & 2 & 2 & 6 \\
\hline
\end{tabular}

Fonte: Própria.

Na Tabela 1 é possível observar que a escolha de uma alternativa que melhor se combina com outras não é nem a primeira (A8), nem a segunda (A5) da ordenação por média ponderada, e sim as terceira (A7) e sexta (A2) opções.

Isto é, apesar de não figurarem na preferência para uma decisão única, estas alternativas figuram nas primeiras posições da ordenação em conjunto. Apesar de não serem as melhores individualmente, estas são potencialmente bons pares para as demais.

\section{CONCLUSÕES}

Este trabalho teve como objetivo apresentar o MEM para escolha dupla, e como já esperado, assim como o MEG, o MEM apresenta um resultado diferente da ordenação por média ponderada, mas acrescenta aos procedimentos internos do algoritmo melhoramentos, estando preparado para situações mais próximas a realidade, interagindo com diferentes escalas de medições e direções diversas de preferência.

Quanto aos resultados, aponta-se que as duas primeiras escolhas resultantes do modelo de média ponderada figuram somente na décima primeira posição do modelo proposto, aquilatando que o melhor grupo de óôalternativas não é necessariamente o grupo das ónô primeiras alternativas em uma ordenação. As alternativas dentro do conjunto de melhores soluções, que figuram empatadas na primeira posição ocupam as posições 4 e 6 (A1 e A2) e posições 1 e 3 (A7 e A8) na ordenação advinda da média ponderada.

Aponta-se ainda que as alternativas A2 e A7 podem ser consideradas mais complementáveis, isto é, são alternativas que melhor complementam as demais dentro do conjunto de alternativas viáveis, e devem ser preferencialmente optadas em caso de escolha única com vistas à escolha dupla.

Para trabalhos futuros, sugere-se utilizar o software apresentado e o algoritmo desenvolvido em problemas de localização estratégica, aonde se deseja alocar com mais de uma unidade em um território, podendo ser a expansão de uma franquia com um número determinado de lojas, ou também a instalação de bases de suprimento ou centro de distribuição. 


\section{REFERÊNCIAS BIBLIOGRÁFICAS}

[1] ALMEIDA, A. T. DE; DUARTE, M. D. O. A multi-criteria decision model for selecting project portfolio with consideration being given to a new concept for synergies. Pesquisa Operacional, v. 31, n. 2, p. 301ї 318, ago. 2011.

[2] BANA E COSTA, C. A. Três convicções fundamentais na prática do apoio à decisão. Pesquisa Operacional, v. 13, n. 1, p. 9ï 20, jun. 1993.

[3] BELTON, V.; STEWART, T. J. Multiple Criteria Decision Analysis: An Integrated Approach. London: Springer, 2002.

[4] BRANS, J. P.; VINCKE, P.; MARESCHAL, B. How to select and how to rank projects: The Promethee method. European Journal of Operational Research, v. 24, n. 2, p. 228 ï 238, Fevereiro 1986.

[5] DEMING, W. E. Qualidade: a revolução da administração. Tradução Francis Henrik Aubert; Maria Esmene Comenale; Aurea Consenza Dal Bó. Rio de Janeiro: MarquesSaraiva, 1990.

[6] EHRGOTT, M. Multicriteria optimization. 2. ed. New Zeland: Springer Verlag, 2005. v. 491

[7] FAÇANHA, S. L. DE O.; YU, A. S. O. Abordagem integrada. In: YU, A. S. O. (Ed.). Tomada de decisão nas organizações. São Paulo: Saraiva, 2011. p. 511 75.

[8] FIGUEIRA, J. R. M.; MOUSSEAU, V.; ROY, B. ELECTRE methods. In: FIGUEIRA, J. R. M.; GRECO, S.; EHRGOTT, M. (Eds.). Multiple Criteria Decision Analysis: State Of The Art Surveys. London: Kluwer Academic Publishers, 2005. p. 1331162.

[9] GOMES, L. F. A. M.; GONZÁLEZ-ARAYA, M. C.; CARIGNANO, C. Tomada de decisões em cenários complexos: introdução aos modelos discretos de apoio multicritério à decisão. São Paulo: Thomson, 2004.

[10] GOMES, L. F. A. M.; LIMA, M. M. P. P. TODIM: Basics and application to multicriteria ranking of projects with environmental impacts. Foundations of Control Engineering, v. Vol. 16, n. No. 3-4, p. 113ї 127, 1991.

[11] GRAVETTER, F. J.; WALLNAU, L. B. Statistics for the Behavioral Sciences. Belmont: Cengage Learning, 2008.

[12] HORA, H. R. M. DA et al. MEG - Proposta de um método multicritério de seleção dupla. XVI CLAIO/XLIV SBPO. Anais... In: XVI CLAIO/XLIV SBPO. Rio de Janeiro: SOBRAPO, 2012

[13] HORA, H. R. M.; COSTA, H. G. MEG ï Double Selection Multicriteria Method Proposal. ISERC. Anais... In: INDUSTRIAL AND SYSTEM ENGINEERING RESEARCH CONFERENCE. Puerto Rico: IIE, 2013

[14] MARICHAL, J.-L.; ROUBENS, M. Determination of weights of interacting criteria from a reference set. European Journal of Operational Research, v. 124, n. 3, p. 641ї 650, Agosto 2000.

[15] PAGANO, M.; GAUVREAU, K. Princípios de bioestatística. Tradução Sérgio de Castro Paiva. 1. ed. São Paulo: Thomson, 2004.

[16] RAIFFA, H. Decision Analysis: A Personal Account of How It Got Started and Evolved. Operations Research, v. 50, n. 1, p. 179ї 185, 1 jan. 2002. 
[17] ROGERS, M.; BRUEN, M.; MAYSTRE, L.-Y. Electre and decision support [t: methods and applications in engineering and infrastructure investment. Boston: Kluwer Academic, 2000.

[18] ROY, B.; BOUYSSOU, D. Aide Multicritère à la décision: méthodes et cas. Paris: Economica, 1993.

[19] SAATY, T. L. A scaling method for priorities in hierarchical structures. Journal of Mathematical Psychology, v. 15, n. 3, p. 234ї 281, jun. 1977.

[20] TANINO, T. Sensitive Analysis in MCDM. In: GÁL, T.; STEWART, T. J.; HANNE, T. (Eds.). Multicriteria Decision Making: Advances in MCDM Models, Algorithms, Theory, and Applications. London: Springer, 1999. p. 7.1 ï 7.29.

[21] TIETZ, R. Semi-normative theories based on bounded rationality. Journal of Economic Psychology, v. 13, n. 2, p. 297ï 314, jun. 1992.

[22] VARGAS, L. G. An overview of the analytic hierarchy process and its applications. European Journal of Operational Research, v. 48, n. 1, p. 2ï 8, 5 set. 1990.

[23] VINCKE, P. Multicriteria decision-aid. Tradução Marjorie Gassner. Chichester, England: Wiley, 1992. 